\title{
Oscillation criteria for certain second-order Emden-Fowler delay functional dynamic equations with damping on time scales
}

\author{
Jiashan Yang* and Xuewen Qin
}

"Correspondence: syxyyjs@163.com School of Information and Electronic Engineering, Wuzhou University, Wuzhou, Guangxi 543002, China

\section{照 Springer}

\begin{abstract}
This paper is concerned with oscillations of certain second-order Emden-Fowler variable delay functional dynamic equations with damping and neutral of the form$$
\left[A(t) \varphi\left(y^{\Delta}(t)\right)\right]^{\Delta}+b(t) \varphi\left(y^{\Delta}(t)\right)+P(t) F(\varphi(x(\delta(t))))-Q(t) f(\varphi(x(\gamma(t))))=0
$$

on an arbitrary time scale $\mathbf{T}$, where $y(t)=x(t)+B(t) x(\tau(t))$ and $\varphi(u)=|u|^{\lambda-1} u(\lambda>0)$. By using the generalized Riccati transformation and the inequality technique, some new oscillation criteria for the equations are established. Our results extend and improve some known results, but they also unify the oscillation of second-order Emden-Fowler delay differential equations with damping and second-order Emden-Fowler delay difference equations with damping. Examples are given to illustrate the importance of our results.
\end{abstract}

MSC: 34K11;34C10;39A10

Keywords: oscillation; delay dynamic equations; Riccati transformation; damping

\section{Introduction}

The theory of time scales, which has recently received a lot of attention, was introduced in [1], in order to unify continuous and discrete analysis. Several authors have expounded on various aspects of this new theory; see [2-6]. A time scale $\mathbf{T}$ is an arbitrary closed subset of the reals, and the cases when this time scale is equal to the reals or to the integers represent the classical theories of differential and of difference equations. Many other interesting time scales exist, and they give rise to plenty of applications, among them the study of discrete dynamic models of populations which are in season (and may follow a difference scheme with variable step size but are often modeled by continuous dynamic systems), die out, say in winter, while their eggs are incubating or dormant, and then in season again, and hatching gives rise to a nonoverlapping population (see [4]). Not only does the new theory of so-called 'dynamic equations' unify the theories of differential equations and difference equations, but also it extends these classical cases to cases 'in between', e.g., to so-called $q$-difference equations when $\mathbf{T}=q^{\mathbf{N}_{0}}=\left\{q^{t}: t \in \mathbf{N}_{0}\right\}$ for some $q>1$ (which has important applications in quantum theory) and can be applied on different time scales like $\mathbf{T}=h \mathbf{N}, \mathbf{T}=\mathbf{N}^{2}=\left\{t^{2}: t \in \mathbf{N}\right\}, \mathbf{T}=\mathbf{T}_{n}=\left\{t_{n}=\sum_{k=1}^{n} \frac{1}{k}, n \in \mathbf{N}_{0}\right\}$ and the space of the harmonic numbers.

() 2015 Yang and Qin; licensee Springer. This is an Open Access article distributed under the terms of the Creative Commons Attribution License (http://creativecommons.org/licenses/by/4.0), which permits unrestricted use, distribution, and reproduction in any medium, provided the original work is properly credited. 
In recent years, there has been much research activity concerning the oscillation and nonoscillation of solutions of various equations on time scales, and we refer the reader to the studies in [7-9]. However, there are few results dealing with the oscillation of the solutions of second-order delay dynamic equations with damping on time scales [10-28]. In this article, we study oscillatory behavior of all solutions of second-order Emden-Fowler variable delay functional dynamic equations with damping and neutral

$$
\begin{aligned}
& {\left[A(t) \varphi\left(y^{\Delta}(t)\right)\right]^{\Delta}+b(t) \varphi\left(y^{\Delta}(t)\right)+P(t) F(\varphi(x(\delta(t))))-Q(t) f(\varphi(x(\gamma(t))))=0,} \\
& \quad t \in \mathbf{T}, t \geq t_{0},
\end{aligned}
$$

where $y(t)=x(t)+B(t) x(\tau(t)), \varphi(u)=|u|^{\lambda-1} u, \lambda>0$, subject to the following hypotheses:

$\left(\mathrm{H}_{1}\right) \mathbf{T}$ is a time scale (i.e., a nonempty closed subset of the real numbers $\mathbf{R}$ ) which is unbounded above, and $t_{0} \in \mathbf{T}$ with $t_{0}>0$, we define a time scale interval of the form $\left[t_{0},+\infty\right)_{\mathbf{T}}$ by $\left[t_{0},+\infty\right)_{\mathbf{T}}=\left[t_{0},+\infty\right) \cap \mathbf{T}$. $A(t), B(t), b(t), P(t), Q(t) \in C_{r d}(\mathbf{T}, \mathbf{R})$, i.e., $A(t), B(t), b(t), P(t), Q(t): \mathbf{T} \rightarrow \mathbf{R}$ are rd-continuous functions. $F(u), f(u): \mathbf{R} \rightarrow \mathbf{R}$ are continuous functions with $u F(u)>0(u \neq 0)$ and $u f(u)>0(u \neq 0)$.

$\left(\mathrm{H}_{2}\right) \tau(t), \delta(t), \gamma(t): \mathbf{T} \rightarrow \mathbf{T}$ are delay functions such that $\tau(t) \leq t, \lim _{t \rightarrow+\infty} \tau(t)=+\infty$; $\gamma(t)=\delta(t) \leq t, \lim _{t \rightarrow+\infty} \delta(t)=+\infty$.

$\left(\mathrm{H}_{3}\right) \quad 0 \leq B(t)<1 ; b(t) \geq 0 ; P(t) \geq 0 ; Q(t) \geq 0 ; A(t)>0, A^{\Delta}(t) \geq 0$ and $-b / A \in \mathfrak{R}^{+}$.

$\left(\mathrm{H}_{4}\right)$ There exist constants $L>0$ and $\eta>0$ such that $F(u) / u \geq L(u \neq 0), f(u) / u \leq \eta(u \neq 0)$ and $\Phi(t)=L P(t)-\eta Q(t)>0$.

By a solution of (1.1), we mean a nontrivial real-valued function $x(t)$ satisfying (1.1) for $t \in \mathbf{T}$. We recall that a solution $x(t)$ of $(1.1)$ is said to be oscillatory on $\left[t_{0},+\infty\right)_{\mathbf{T}}$ if it is neither eventually positive nor eventually negative; otherwise, the solution is said to be nonoscillatory. Equation (1.1) is said to be oscillatory if all of its solutions are oscillatory. Our attention is restricted to those solutions $x(t)$ of $(1.1)$ where $x(t)$ is not eventually identically zero.

Note that if $\mathbf{T}=\mathbf{R}$, then $\sigma(t)=t, \mu(t)=0, x^{\Delta}(t)=x^{\prime}(t)$, and (1.1) becomes second-order differential equation

$$
\begin{aligned}
& {\left[A(t) \varphi\left(y^{\prime}(t)\right)\right]^{\prime}+b(t) \varphi\left(y^{\prime}(t)\right)+P(t) F(\varphi(x(\delta(t))))-Q(t) f(\varphi(x(\gamma(t))))=0,} \\
& \quad t \in \mathbf{R} .
\end{aligned}
$$

If $\mathbf{T}=\mathbf{Z}$, then $\sigma(t)=t+1, \mu(t)=1, x^{\Delta}(t)=\Delta x(t)=x(t+1)-x(t)$, and (1.1) becomes secondorder difference equation

$$
\begin{aligned}
& \Delta[A(t) \varphi(\Delta y(t))]+b(t) \varphi(\Delta y(t))+P(t) F(\varphi(x(\delta(t))))-Q(t) f(\varphi(x(\gamma(t))))=0, \\
& \quad t \in \mathbf{Z} .
\end{aligned}
$$

If $\mathbf{T}=q^{\mathbf{N}_{0}}=\left\{q^{t}: t \in \mathbf{N}_{0}, q>1\right\}$, then $\sigma(t)=q t, \mu(t)=(q-1) t, x^{\Delta}(t)=\Delta_{q} x(t)=\frac{x(q t)-x(t)}{(q-1) t}$, and (1.1) becomes a second-order $q$-difference equation

$$
\Delta_{q}\left[A(t) \varphi\left(\Delta_{q} y(t)\right)\right]+b(t) \varphi\left(\Delta_{q} y(t)\right)+P(t) F(\varphi(x(\delta(t))))-Q(t) f(\varphi(x(\gamma(t))))=0 .
$$


In (1.1), if $\lambda=1, Q(t) \equiv 0$, then (1.1) is simplified to the equation

$$
\begin{aligned}
& \left\{A(t)[x(t)+B(t) x(\tau(t))]^{\Delta}\right\}^{\Delta}+b(t)[x(t)+B(t) x(\tau(t))]+P(t) F(x(\delta(t)))=0, \\
& \quad t \in \mathbf{T}, t \geq t_{0} .
\end{aligned}
$$

If $B(t) \equiv 0$ and $Q(t) \equiv 0$ in (1.1), then (1.1) is simplified to the equation

$$
\begin{aligned}
& {\left[A(t)\left|x^{\Delta}(t)\right|^{\lambda-1} x^{\Delta}(t)\right]^{\Delta}+b(t)\left|x^{\Delta}(t)\right|^{\lambda-1} x^{\Delta}(t)+P(t)|x(\delta(t))|^{\lambda-1} x(\delta(t))=0,} \\
& \quad t \in \mathbf{T}, t \geq t_{0} .
\end{aligned}
$$

In (1.5), if $b(t) \equiv 0$ and $B(t) \equiv 0$, then (1.5) is simplified to the equation

$$
\left[A(t) x^{\Delta}(t)\right]^{\Delta}+P(t) F(x(\delta(t)))=0, \quad t \in \mathbf{T}, t \geq t_{0} .
$$

In (1.1), if $B(t) \equiv 0, b(t) \equiv 0, F(u)=u, \delta(t)=t, Q(t) \equiv 0$, and $\lambda$ is an odd, then (1.1) is simplified to the equation

$$
\left\{A(t)\left[x^{\Delta}(t)\right]^{\lambda-1}\right\}^{\Delta}+P(t)[x(t)]^{\lambda-1}=0, \quad t \in \mathbf{T}, t \geq t_{0} .
$$

In (1.5), if $b(t) \equiv 0, B(t) \equiv 0$ and $A(t) \equiv 1$, then (1.5) is simplified to the equation

$$
x^{\Delta \Delta}(t)+P(t) F(x(\delta(t)))=0, \quad t \in \mathbf{T}, t \geq t_{0}
$$

In recent years, there has been an increasing interest in studying the oscillatory behavior of first and second-order neutral delay dynamic equations on time scales, see [1-26]. As a special case of (1.1), Agarwal et al. [12], Sahiner [13] considered second-order delay dynamic equation (1.9), and Saker [14] considered second-order half-linear dynamic equation (1.8), and established some sufficient conditions for oscillation of (1.8) and (1.9). Erbe et al. [16] considered the general nonlinear delay dynamic equation (1.7), setting out to obtain some new oscillation criteria which improve the results given in [13]. So far, oscillation of the second-order nonlinear delay dynamic equation with damping (1.5) has rarely been discussed. Sun et al. [10] extended and improved the results of [12-16], meanwhile obtaining some oscillatory criteria. On this basis, by using the Riccati transformation technique and inequalities, Zhang et al. [21, 22, 25] studied the oscillatory behavior of all solutions of (1.6). Note that the results in [21,22, 25] are based on the condition $\delta(\mathbf{T})=\mathbf{T}$, which can be a restrictive condition and it is not easy to satisfy. For instance, when $\mathbf{T}=\{1,3,5,7, \ldots\}$ and letting $\delta(t)=t-1$, then $\delta$ is a strictly increasing function, $\delta(t) \leq t$ and $\lim _{t \rightarrow+\infty} \delta(t)=+\infty$, but $\delta(\mathbf{T})=\{0,2,4,6, \ldots\} \neq \mathbf{T}$, so the condition $\delta(\mathbf{T})=\mathbf{T}$ in $[21,22,25]$ does not hold and the results in $[21,22,25]$ may not be true.

Hence, it would be interesting to study the oscillation behavior of (1.1) when $\delta(\mathbf{T})=\mathbf{T}$ does not hold. In this paper, we discuss the oscillation of solutions of (1.1). By using the generalized Riccati transformation and the inequality technique, we obtain some new oscillation criteria for (1.1), some results of $[10-17,21,22,25,28]$ are now special examples of our results. We shall also consider the two cases

$$
\int_{t_{0}}^{+\infty}\left[\frac{e_{-b / A}\left(s, t_{0}\right)}{A(s)}\right]^{1 / \lambda} \Delta s=+\infty
$$


and

$$
\int_{t_{0}}^{+\infty}\left[\frac{e_{-b / A}\left(s, t_{0}\right)}{A(s)}\right]^{1 / \lambda} \Delta s<+\infty
$$

\section{Preliminaries}

We shall employ the following lemmas.

Lemma 2.1 [4] Assume that $x(t)$ is $\Delta$-differentiable and eventually positive or eventually negative, then

$$
\left[x^{\lambda}(t)\right]^{\Delta}=\lambda \int_{0}^{1}\left[h x^{\sigma}+(1-h) x\right]^{\lambda-1} x^{\Delta}(t) d h .
$$

Lemma 2.2 [11] Assume that

(i) $u \in C_{r d}^{2}(\mathbf{I}, \mathbf{R})$, where $\mathbf{I}=\left[t^{*},+\infty\right), t^{*}>0$.

(ii) $u(t)>0, u^{\Delta}(t)>0, u^{\Delta \Delta}(t) \leq 0, t \geq t^{*}$.

Then, for every $c \in(0,1)$, there exists a constant $t_{c} \in \mathbf{T}, t_{c}>t^{*}$ such that $u(\sigma(t)) \leq \frac{\sigma(t) u(\delta(t))}{c \delta(t)}$ for all $t \geq t_{c}$.

Lemma 2.3 [4] If $g \in \mathfrak{R}^{+}$, i.e., $g: \mathbf{T} \rightarrow \mathbf{R}$ is $r d$-continuous and such that $1+\mu(t) g(t)>0$ for all $t \in\left[t_{0},+\infty\right)_{\mathbf{T}}$, then the initial value problem $y^{\Delta}(t)=g(t) y(t), y\left(t_{0}\right)=y_{0} \in \mathbf{R}$ has a unique and positive solution on $\left[t_{0},+\infty\right)_{\mathrm{T}}$, denoted by $e_{g}\left(t, t_{0}\right)$. This 'exponential function' satisfies the semigroup property $e_{g}(a, b) e_{g}(b, c)=e_{g}(a, c)$.

Lemma 2.4 [18] Assume that $a$ and $b$ are nonnegative real numbers, then $r a b^{r-1}-a^{r} \leq$ $(r-1) b^{r}$ for all $r>1$, where the equality holds if and only if $a=b$.

Lemma 2.5 ([19], Hölder's inequality) Let $a, b \in \mathbf{T}$ and $a<b$. For $r d$-continuous functions $f, g:[a, b] \rightarrow \mathbf{R}$, we have $\int_{a}^{b}|f(u) g(u)| \Delta u \leq\left(\int_{a}^{b}|f(u)|^{p} \Delta u\right)^{1 / p}\left(\int_{a}^{b}|g(u)|^{q} \Delta u\right)^{1 / q}$, where $p>1$ and $\frac{1}{p}+\frac{1}{q}=1$.

Lemma 2.6 Assume $\left(\mathrm{H}_{1}\right)-\left(\mathrm{H}_{4}\right)$ and (1.10). Let $x(t)$ be an eventually positive solution of $(1.1)$. Then there exists $t_{1} \in\left[t_{0},+\infty\right)_{\mathrm{T}}$ such that

$$
\begin{aligned}
& y(t)>0, \quad y^{\Delta}(t)>0, \quad A(t) \varphi\left(y^{\Delta}(t)\right)>0, \\
& {\left[A(t) \varphi\left(y^{\Delta}(t)\right)\right]^{\Delta} \leq 0 \quad \text { and } \quad x(t) \geq[1-B(t)] y(t)}
\end{aligned}
$$

for all $t \in\left[t_{1},+\infty\right)_{\mathbf{T}}$.

Proof Since $x(t)$ is an eventually positive solution of (1.1), there exists $t_{1} \in\left[t_{0},+\infty\right)_{\mathrm{T}}$ such that $x(t)>0, x(\tau(t))>0, x(\gamma(t))=x(\delta(t))>0$ for all $t \in\left[t_{1},+\infty\right)_{\mathrm{T}}$, thus, $y(t)>0$. From (1.1) we obtain

$$
\begin{aligned}
& {\left[A(t) \varphi\left(y^{\Delta}(t)\right)\right]^{\Delta}+b(t) \varphi\left(y^{\Delta}(t)\right)} \\
& \quad \leq-L P(t)[x(\delta(t))]^{\lambda}+\eta Q(t)[x(\gamma(t))]^{\lambda} \\
& \quad=-[L P(t)-\eta Q(t)][x(\delta(t))]^{\lambda}=-\Phi(t)[x(\delta(t))]^{\lambda}<0, \quad t \in\left[t_{1},+\infty\right)_{\mathbf{T}} .
\end{aligned}
$$


Thus, by Lemma 2.3 , we obtain on $\left[t_{1},+\infty\right)_{\mathrm{T}}$,

$$
\begin{aligned}
{\left[\frac{A(t) \varphi\left(y^{\Delta}(t)\right)}{e_{-b / A}\left(t, t_{0}\right)}\right]^{\Delta} } & =\frac{\left[A(t) \varphi\left(y^{\Delta}(t)\right)\right]^{\Delta} e_{-b / A}\left(t, t_{0}\right)-A(t) \varphi\left(y^{\Delta}(t)\right)\left[e_{-b / A}\left(t, t_{0}\right)\right]^{\Delta}}{e_{-b / A}\left(t, t_{0}\right) e_{-b / A}\left(\sigma(t), t_{0}\right)} \\
& =\frac{\left[A(t) \varphi\left(y^{\Delta}(t)\right)\right]^{\Delta}+b(t) \varphi\left(y^{\Delta}(t)\right)}{e_{-b / A}\left(\sigma(t), t_{0}\right)} \leq-\frac{\Phi(t)[x(\delta(t))]^{\lambda}}{e_{-b / A}\left(\sigma(t), t_{0}\right)}<0 .
\end{aligned}
$$

Hence, $\frac{A(t) \varphi\left(y^{\Delta}(t)\right)}{e_{-b / A}\left(t, t_{0}\right)}=\frac{A(t)\left|y^{\Delta}(t)\right|^{\lambda-1} y^{\Delta}(t)}{e_{-b / A}\left(t, t_{0}\right)}$ is decreasing and, therefore, eventually of one sign, so (use Lemma 2.3) $y^{\Delta}(t)$ is either eventually positive or eventually negative. We assert that $y^{\Delta}(t)>0$ for all $t \in\left[t_{1},+\infty\right)_{\mathrm{T}}$. Assume that $y^{\Delta}(t)<0$ eventually; then there exists $t_{2} \in\left[t_{1},+\infty\right)_{\mathrm{T}}$ such that $y^{\Delta}(t)<0$ for all $t \in\left[t_{2},+\infty\right)_{\mathrm{T}}$. From Lemma 2.3 we obtain

$$
\frac{A(t) \varphi\left(y^{\Delta}(t)\right)}{e_{-b / A}\left(t, t_{0}\right)} \leq \frac{A\left(t_{2}\right) \varphi\left(y^{\Delta}\left(t_{2}\right)\right)}{e_{-b / A}\left(t_{2}, t_{0}\right)}=-M<0 \quad \text { for all } t \in\left[t_{2},+\infty\right)_{\mathrm{T}}
$$

where $M=-\frac{A\left(t_{2}\right) \varphi\left(y^{\Delta}\left(t_{2}\right)\right)}{e_{-b / A}\left(t_{2}, t_{0}\right)}=\frac{A\left(t_{2}\right)\left|y^{\Delta}\left(t_{2}\right)\right|^{\lambda-1}\left[-y^{\Delta}\left(t_{2}\right)\right]}{e_{-b / A}\left(t_{2}, t_{0}\right)}>0$. By the above inequality we obtain $A(t) \varphi\left(y^{\Delta}(t)\right) \leq-M e_{-b / A}\left(t, t_{0}\right)$, therefore, it follows that $\left[-y^{\Delta}(t)\right]^{\lambda} \geq \frac{M e_{-b / A}\left(t, t_{0}\right)}{A(t)}$, i.e.,

$$
y^{\Delta}(t) \leq-M^{\frac{1}{\lambda}}\left[\frac{e_{-b / A}\left(t, t_{0}\right)}{A(t)}\right]^{1 / \lambda} \text { for all } t \in\left[t_{2},+\infty\right)_{\mathrm{T}}
$$

Integrating the above inequality from $t_{2}$ to $t\left(t \in\left[t_{2},+\infty\right)_{\mathrm{T}}\right)$ leads to

$$
y(t) \leq y\left(t_{2}\right)-M^{\frac{1}{\lambda}} \int_{t_{2}}^{t}\left[\frac{e_{-b / A}\left(s, t_{0}\right)}{A(s)}\right]^{1 / \lambda} \Delta s \rightarrow-\infty \quad \text { as } t \rightarrow+\infty,
$$

which contradicts with $y(t)>0$. So $y^{\Delta}(t)>0$, and then $A(t) \varphi\left(y^{\Delta}(t)\right)>0$. From (2.2) we obtain $\left[A(t) \varphi\left(y^{\Delta}(t)\right)\right]^{\Delta} \leq 0$.

Moreover, in view of $x(t) \leq y(t)$, we have

$$
y(t)=x(t)+B(t) x(\tau(t)) \leq x(t)+B(t) y(\tau(t)) \leq x(t)+B(t) y(t)
$$

i.e., $x(t) \geq[1-B(t)] y(t)$. This completes the proof.

\section{Main results}

In this section, we use the generalized Riccati transformation and the inequality technique to obtain some sufficient conditions for oscillation of all solutions of (1.1), provided the condition (1.10) holds. If (1.10) is not satisfied, i.e., if (1.11) holds, then we present some conditions that guarantee that each solution of (1.1) is either oscillatory or converges to zero.

For convenience, consider the set $D=\left\{(t, s): t \geq s \geq t_{0}, t, s \in\left[t_{0},+\infty\right)_{\mathrm{T}}\right\}$. We say that a function $H=H(t, s)$ belongs to function class $\Omega$, denoted by $H \in \Omega$, if $H \in C_{r d}(D, \mathbf{R})$, which satisfies

$$
H(t, t)=0 \quad \text { for } t \geq t_{0}, \quad H(t, s)>0 \quad \text { for } t>s \geq t_{0}, t, s \in\left[t_{0},+\infty\right)_{\mathrm{T}}
$$

and has a nonpositive continuous $\Delta$-partial derivative $H_{s}^{\Delta}(t, s)$ with respect to the second variable, i.e., $H_{s}^{\Delta}(t, s) \in C_{r d}$ and $H_{s}^{\Delta}(t, s) \leq 0$. 
Theorem 3.1 Assume $\left(\mathrm{H}_{1}\right)-\left(\mathrm{H}_{4}\right)$ and (1.10). If there exist $H \in \Omega$ and a positive and $\Delta$ differentiable function $\phi: \mathbf{T} \rightarrow \mathbf{R}$ such that

$$
\limsup _{t \rightarrow+\infty} \frac{1}{H\left(t, t_{0}\right)} \int_{T_{0}}^{t}\left\{c^{\lambda} H(t, s) \phi(s) \Psi(s)-\frac{[|h(t, s)| \phi(\sigma(s)) A(\sigma(s))]^{\lambda+1}}{(\lambda+1)^{\lambda+1}[\phi(s) A(s) H(t, s)]^{\lambda}}\right\} \Delta s=+\infty
$$

for some constant $T_{0} \geq t_{0}$, where the constant $c$ is defined as in Lemma 2.2, the functions $\Psi(s)$ and $h(t, s)$ are defined as follows:

$$
\begin{aligned}
& \Psi(s)=\Phi(s)[1-B(\delta(s))]^{\lambda}\left[\frac{\delta(s)}{\sigma(s)}\right]^{\lambda}, \\
& h(t, s)=[H(t, s)]_{s}^{\Delta}+\left[\phi^{\Delta}(s)-\frac{\phi(s) b(s)}{A(\sigma(s))}\right] \frac{H(t, s)}{\phi(\sigma(s))} .
\end{aligned}
$$

Then (1.1) is oscillatory on $\left[t_{0},+\infty\right)_{\mathrm{T}}$.

Proof Suppose that (1.1) has a nonoscillatory solution $x(t)$ on $\left[t_{0},+\infty\right)_{\mathrm{T}}$. We may assume without loss of generality that $x(t)>0$ and $x(\tau(t))>0, x(\delta(t))>0$ for all $t \in\left[t_{1},+\infty\right)_{\mathrm{T}}$, $t_{1} \in\left[t_{0},+\infty\right)_{\mathrm{T}}$. Then $y(t)>0$. Now, we claim that

$$
y^{\Delta \Delta}(t) \leq 0 \quad \text { and } \quad \begin{cases}{\left[(y(t))^{\lambda}\right]^{\Delta} \geq \lambda(y(t))^{\lambda-1} y^{\Delta}(t),} & \text { if } \lambda \geq 1, \\ {\left[(y(t))^{\lambda}\right]^{\Delta} \geq \lambda[y(\sigma(t))]^{\lambda-1} y^{\Delta}(t),} & \text { if } 0<\lambda<1 .\end{cases}
$$

In fact, if $\lambda \geq 1$, then, by (2.1), we get

$$
\left[(y(t))^{\lambda}\right]^{\Delta} \geq \lambda \int_{0}^{1}[h y+(1-h) y]^{\lambda-1} y^{\Delta}(t) d h=\lambda(y(t))^{\lambda-1} y^{\Delta}(t),
$$

and then $\left[\left(y^{\Delta}(t)\right)^{\lambda}\right]^{\Delta} \geq \lambda\left(y^{\Delta}(t)\right)^{\lambda-1} y^{\Delta \Delta}(t)$. Hence,

$$
\begin{aligned}
{\left[A(t)\left(y^{\Delta}(t)\right)^{\lambda}\right]^{\Delta} } & =A^{\Delta}(t)\left(y^{\Delta}(t)\right)^{\lambda}+A(\sigma(t))\left[\left(y^{\Delta}(t)\right)^{\lambda}\right]^{\Delta} \\
& \geq A^{\Delta}(t)\left(y^{\Delta}(t)\right)^{\lambda}+\lambda A(\sigma(t))\left(y^{\Delta}(t)\right)^{\lambda-1} y^{\Delta \Delta}(t)
\end{aligned}
$$

in view of Lemma 2.6 and $A^{\Delta}(t) \geq 0$, we get $y^{\Delta \Delta}(t) \leq 0$.

If $0<\lambda<1$, then, by (2.1), we get

$$
\left[(y(t))^{\lambda}\right]^{\Delta} \geq \lambda \int_{0}^{1}\left[h y^{\sigma}+(1-h) y^{\sigma}\right]^{\lambda-1} y^{\Delta}(t) d h=\lambda[y(\sigma(t))]^{\lambda-1} y^{\Delta}(t) .
$$

Similarly, we can easily find $y^{\Delta \Delta}(t) \leq 0$, and hence this completes the proof of the claim. By Lemma 2.2, there exists $T_{0} \in\left[t_{1},+\infty\right)_{\mathrm{T}}$ with $T_{0} \geq \max \left\{t_{c}, t_{1}\right\}, \forall c \in(0,1)$, we have

$$
y(\delta(t)) \geq \frac{c \delta(t)}{\sigma(t)} y(\sigma(t)) \geq \frac{c \delta(t)}{\sigma(t)} y(t) \quad \text { for all } t \in\left[T_{0},+\infty\right)_{\mathrm{T}} .
$$

Now define the function $w(t)$ by

$$
w(t)=\phi(t) \frac{A(t) \varphi\left(y^{\Delta}(t)\right)}{\varphi(y(t))}=\phi(t) \frac{A(t)\left(y^{\Delta}(t)\right)^{\lambda}}{(y(t))^{\lambda}} \quad \text { on }\left[T_{0},+\infty\right)_{\mathrm{T}} .
$$

Then we have $w(t)>0\left(t \in\left[T_{0},+\infty\right)_{\mathrm{T}}\right)$. 
If $\lambda \geq 1$, then, by (3.5) (use (2.2), Lemma 2.6 and $\left[(y(t))^{\lambda}\right]^{\Delta} \geq \lambda(y(t))^{\lambda-1} y^{\Delta}(t)$ ), we obtain for $t \in\left[T_{0},+\infty\right)_{\mathbf{T}}$

$$
\begin{aligned}
w^{\Delta}(t)= & \phi^{\Delta}(t) \frac{A(\sigma(t)) \varphi\left(y^{\Delta}(\sigma(t))\right)}{\varphi(y(\sigma(t)))} \\
& +\phi(t) \frac{\left[A(t)\left(y^{\Delta}(t)\right)^{\lambda}\right]^{\Delta}(y(t))^{\lambda}-A(t)\left(y^{\Delta}(t)\right)^{\lambda}\left[(y(t))^{\lambda}\right]^{\Delta}}{(y(t))^{\lambda}[y(\sigma(t))]^{\lambda}} \\
\leq & \frac{\phi^{\Delta}(t) w(\sigma(t))}{\phi(\sigma(t))}-\phi(t) \frac{\Phi(t)[x(\delta(t))]^{\lambda}+b(t) \varphi\left(y^{\Delta}(t)\right)}{[y(\sigma(t))]^{\lambda}}-\phi(t) \frac{A(t)\left(y^{\Delta}(t)\right)^{\lambda}\left[(y(t))^{\lambda}\right]^{\Delta}}{(y(t))^{\lambda}[y(\sigma(t))]^{\lambda}} \\
\leq & \frac{\phi^{\Delta}(t) w(\sigma(t))}{\phi(\sigma(t))}-\phi(t) \Phi(t)[1-B(\delta(t))]^{\lambda} \frac{[y(\delta(t))]^{\lambda}}{[y(\sigma(t))]^{\lambda}} \\
& -\phi(t) \frac{b(t)\left[y^{\Delta}(t)\right]^{\lambda}}{[y(\sigma(t))]^{\lambda}}-\lambda \phi(t) \frac{A(t)\left(y^{\Delta}(t)\right)^{\lambda+1}}{y(t)[y(\sigma(t))]^{\lambda}} .
\end{aligned}
$$

From $y^{\Delta \Delta}(t) \leq 0$ and $y^{\Delta}(t)>0$, we get $y^{\Delta}(t) \geq y^{\Delta}(\sigma(t))$ and $y(t) \leq y(\sigma(t))$. In view of the first formula of (3.2) and (3.4), it follows from (3.6) that

$$
\begin{aligned}
w^{\Delta}(t) \leq & \frac{\phi^{\Delta}(t) w(\sigma(t))}{\phi(\sigma(t))}-\phi(t) \Phi(t)[1-B(\delta(t))]^{\lambda}\left[\frac{c \delta(t)}{\sigma(t)}\right]^{\lambda} \\
& -\phi(t) \frac{b(t)\left[y^{\Delta}(\sigma(t)]^{\lambda}\right.}{[y(\sigma(t))]^{\lambda}}-\lambda \phi(t) \frac{A(t)\left[y^{\Delta}(\sigma(t))\right]^{\lambda+1}}{[y(\sigma(t))]^{\lambda+1}} \\
= & {\left[\frac{\phi^{\Delta}(t)}{\phi(\sigma(t))}-\frac{\phi(t) b(t)}{\phi(\sigma(t)) A(\sigma(t))}\right] w(\sigma(t))-c^{\lambda} \phi(t) \Psi(t) } \\
& -\frac{\lambda \phi(t) A(t)[w(\sigma(t))]^{\frac{\lambda+1}{\lambda}}}{[\phi(\sigma(t)) A(\sigma(t))]^{\frac{\lambda+1}{\lambda}}} \text { for } t \in\left[T_{0},+\infty\right)_{\mathrm{T}},
\end{aligned}
$$

i.e.,

$$
\begin{aligned}
c^{\lambda} \phi(t) \Psi(t) \leq & -w^{\Delta}(t)+\left[\phi^{\Delta}(t)-\frac{\phi(t) b(t)}{A(\sigma(t))}\right] \frac{w(\sigma(t))}{\phi(\sigma(t))} \\
& -\lambda \phi(t) A(t)\left[\frac{w(\sigma(t))}{\phi(\sigma(t)) A(\sigma(t))}\right]^{\frac{\lambda+1}{\lambda}} \text { for } t \in\left[T_{0},+\infty\right)_{\mathbf{T}} .
\end{aligned}
$$

If $0<\lambda<1$, in view of $\left[(y(t))^{\lambda}\right]^{\Delta} \geq \lambda[y(\sigma(t))]^{\lambda-1} y^{\Delta}(t)$, then (3.6) becomes

$$
\begin{aligned}
w^{\Delta}(t) \leq & \frac{\phi^{\Delta}(t) w(\sigma(t))}{\phi(\sigma(t))}-\phi(t) \Phi(t)[1-B(\delta(t))]^{\lambda} \frac{[y(\delta(t))]^{\lambda}}{[y(\sigma(t))]^{\lambda}} \\
& -\phi(t) \frac{b(t)\left[y^{\Delta}(t)\right]^{\lambda}}{[y(\sigma(t))]^{\lambda}}-\lambda \phi(t) \frac{A(t)\left(y^{\Delta}(t)\right)^{\lambda+1}}{(y(t))^{\lambda} y(\sigma(t))}
\end{aligned}
$$

similarly, we can get (3.7). Then from (3.7), we can obtain

$$
\begin{aligned}
& \int_{T_{0}}^{t} c^{\lambda} H(t, s) \phi(s) \Psi(s) \Delta s \\
& \quad \leq-\int_{T_{0}}^{t} H(t, s) w^{\Delta}(s) \Delta s+\int_{T_{0}}^{t} H(t, s)\left[\phi^{\Delta}(s)-\frac{\phi(s) b(s)}{A(\sigma(s))}\right] \frac{w(\sigma(s))}{\phi(\sigma(s))} \Delta s
\end{aligned}
$$




$$
\begin{aligned}
& -\int_{T_{0}}^{t} H(t, s) \frac{\lambda \phi(s) A(s)}{[\phi(\sigma(s)) A(\sigma(s))]^{\frac{\lambda+1}{\lambda}}}[w(\sigma(s))]^{\frac{\lambda+1}{\lambda}} \Delta s \\
= & -[H(t, s) w(s)]_{T_{0}}^{t}+\int_{T_{0}}^{t}[H(t, s)]_{s}^{\Delta} w(\sigma(s)) \Delta s \\
& +\int_{T_{0}}^{t} H(t, s)\left[\phi^{\Delta}(s)-\frac{\phi(s) b(s)}{A(\sigma(s))}\right] \frac{w(\sigma(s))}{\phi(\sigma(s))} \Delta s \\
& -\int_{T_{0}}^{t} H(t, s) \frac{\lambda \phi(s) A(s)}{[\phi(\sigma(s)) A(\sigma(s))]^{\frac{\lambda+1}{\lambda}}}[w(\sigma(s))]^{\frac{\lambda+1}{\lambda}} \Delta s \\
\leq & H\left(t, T_{0}\right) w\left(T_{0}\right)+\int_{T_{0}}^{t}\left\{[H(t, s)]_{s}^{\Delta}+\left[\phi^{\Delta}(s)-\frac{\phi(s) b(s)}{A(\sigma(s))}\right] \frac{H(t, s)}{\phi(\sigma(s))}\right\} w(\sigma(s)) \Delta s \\
& -\int_{T_{0}}^{t} H(t, s) \frac{\lambda \phi(s) A(s)}{[\phi(\sigma(s)) A(\sigma(s))]^{\frac{\lambda+1}{\lambda}}}[w(\sigma(s))]^{\frac{\lambda+1}{\lambda}} \Delta s \\
= & H\left(t, T_{0}\right) w\left(T_{0}\right)+\int_{T_{0}}^{t} h(t, s) w(\sigma(s)) \Delta s \\
& -\int_{T_{0}}^{t} \frac{\lambda \phi(s) A(s) H(t, s)}{[\phi(\sigma(s)) A(\sigma(s))]^{\frac{\lambda+1}{\lambda}}[w(\sigma(s))]^{\frac{\lambda+1}{\lambda}} \Delta s .}
\end{aligned}
$$

Now, in Lemma 2.4, we let

$$
\begin{aligned}
& r=\frac{\lambda+1}{\lambda}, \quad a=[\lambda \phi(s) A(s) H(t, s)]^{\frac{\lambda}{\lambda+1}} \frac{w(\sigma(s))}{\phi(\sigma(s)) A(\sigma(s))}, \\
& b=\left(\frac{\lambda}{\lambda+1}\right)^{\lambda}[|h(t, s)| \phi(\sigma(s)) A(\sigma(s))]^{\lambda}[\lambda \phi(s) A(s) H(t, s)]^{\frac{\lambda^{2}}{\lambda+1}} .
\end{aligned}
$$

From Lemma 2.4, we then obtain

$$
|h(t, s)| w(\sigma(s))-\frac{\lambda \phi(s) A(s) H(t, s)}{[\phi(\sigma(s)) A(\sigma(s))]^{\frac{\lambda+1}{\lambda}}}[w(\sigma(s))]^{\frac{\lambda+1}{\lambda}} \leq \frac{[|h(t, s)| \phi(\sigma(s)) A(\sigma(s))]^{\lambda+1}}{(\lambda+1)^{\lambda+1}[\phi(s) A(s) H(t, s)]^{\lambda}} .
$$

Hence, (3.8) implies

$$
\int_{T_{0}}^{t} c^{\lambda} H(t, s) \phi(s) \Psi(s) \Delta s \leq H\left(t, T_{0}\right) w\left(T_{0}\right)+\int_{T_{0}}^{t} \frac{[|h(t, s)| \phi(\sigma(s)) A(\sigma(s))]^{\lambda+1}}{(\lambda+1)^{\lambda+1}[\phi(s) A(s) H(t, s)]^{\lambda}} \Delta s,
$$

and therefore

$$
\begin{aligned}
& \int_{T_{0}}^{t}\left\{c^{\lambda} H(t, s) \phi(s) \Psi(s)-\frac{[|h(t, s)| \phi(\sigma(s)) A(\sigma(s))]^{\lambda+1}}{(\lambda+1)^{\lambda+1}[\phi(s) A(s) H(t, s)]^{\lambda}}\right\} \Delta s \\
& \quad \leq H\left(t, T_{0}\right) w\left(T_{0}\right) \leq H\left(t, t_{0}\right) w\left(T_{0}\right) .
\end{aligned}
$$

Thus

$$
\frac{1}{H\left(t, t_{0}\right)} \int_{T_{0}}^{t}\left\{c^{\lambda} H(t, s) \phi(s) \Psi(s)-\frac{[|h(t, s)| \phi(\sigma(s)) A(\sigma(s))]^{\lambda+1}}{(\lambda+1)^{\lambda+1}[\phi(s) A(s) H(t, s)]^{\lambda}}\right\} \Delta s \leq w\left(T_{0}\right),
$$

contradicting (3.1). This completes the proof. 
Remark 3.1 From Theorem 3.1, we can obtain different conditions for oscillation of all solutions of (1.1) with different choices of $H(t, s)$ and $\phi(t)$. For example, $H(t, s)=(t-s)^{\omega}$, $H(t, s)=\left(\ln \frac{t+1}{s+1}\right)^{\omega}$, or $H(t, s)=\left[\int_{s}^{t} u(t) \Delta t\right]^{\omega}(u(t)>0)$, etc. Now, let us consider the function $H(t, s)$ defined by

$$
H(t, s)=(t-s)^{\omega}, \quad \omega \geq 1, t \geq s \geq t_{0}, t, s \in\left[t_{0},+\infty\right)_{\mathbf{T}} .
$$

Then using the same idea as in the proof of Theorem 3.1, we can now obtain the following result.

Theorem 3.2 Assume $\left(\mathrm{H}_{1}\right)-\left(\mathrm{H}_{4}\right)$ and (1.10). If there exists a positive and $\Delta$-differentiable function $\phi: \mathbf{T} \rightarrow \mathbf{R}$ and $\omega \geq 1$ such that

$$
\begin{aligned}
\limsup _{t \rightarrow+\infty} & \frac{1}{t^{\omega}} \int_{T_{0}}^{t}(t-s)^{\omega}\left\{c^{\lambda} \phi(s) \Psi(s)\right. \\
& \left.-\frac{(\lambda+1)^{-(\lambda+1)}}{[\phi(s) A(s)]^{\lambda}}\left[\left|\phi^{\Delta}(s)-\frac{\phi(s) b(s)}{A(\sigma(s))}\right| A(\sigma(s))\right]^{\lambda+1}\right\} \Delta s=+\infty
\end{aligned}
$$

for some constant $T_{0} \geq t_{0}$, where constant $c$ is defined as in Lemma 2.2, $\Psi(s)$ is defined as in Theorem 3.1, then (1.1) is oscillatory on $\left[t_{0},+\infty\right)_{\mathrm{T}}$.

If (3.1) does not hold, then we have the following results.

Theorem 3.3 Assume $\left(\mathrm{H}_{1}\right)-\left(\mathrm{H}_{4}\right)$ and (1.10). If there exist functions $H \in \Omega, \zeta_{1}(t), \zeta_{2}(t) \in$ $C_{r d}(\mathbf{T}, \mathbf{R})$, and a positive, nondecreasing, differentiable function $\phi \in C_{r d}\left(\mathbf{T}, \mathbf{R}^{+}\right)$such that

$$
\begin{aligned}
& \limsup _{t \rightarrow+\infty} \frac{1}{H\left(t, t_{0}\right)} \int_{u}^{t} H(t, s) \phi(s) \Psi(s) \Delta s \geq \zeta_{1}(u), \\
& \limsup _{t \rightarrow+\infty} \frac{1}{H\left(t, t_{0}\right)} \int_{u}^{t} \frac{[|h(t, s)| \phi(\sigma(s)) A(\sigma(s))]^{\lambda+1}}{[\phi(s) A(s) H(t, s)]^{\lambda}} \Delta s \leq \zeta_{2}(u)
\end{aligned}
$$

for $u \geq T_{0}$, and

$$
\liminf _{t \rightarrow+\infty} \frac{1}{H\left(t, t_{0}\right)} \int_{T_{0}}^{t} \frac{H(t, s) \phi(s) A(s)\left[\zeta_{1}(\sigma(s))-\theta \zeta_{2}(\sigma(s))\right]_{+}^{(1+\lambda) / \lambda}}{[\phi(\sigma(s)) A(\sigma(s))]^{(\lambda+1) / \lambda}} \Delta s=+\infty
$$

for some constant $T_{0} \geq t_{0}$, where $\left[\zeta_{1}(\sigma(s))-\theta \zeta_{2}(\sigma(s))\right]_{+}=\max \left\{\left[\zeta_{1}(\sigma(s))-\theta \zeta_{2}(\sigma(s))\right], 0\right\}$, $\theta=c^{-\lambda}(\lambda+1)^{-(\lambda+1)}$, the constant $c$ is defined as in Lemma 2.2, the functions $\Psi(s)$ and $h(t, s)$ are defined as in Theorem 3.1, then (1.1) is oscillatory on $\left[t_{0},+\infty\right)_{\mathrm{T}}$.

Proof Suppose that (1.1) has a nonoscillatory solution $x(t)$ on $\left[t_{0},+\infty\right)_{\mathrm{T}}$. We may assume without loss of generality that $x(t)>0$ and $x(\tau(t))>0, x(\delta(t))>0$ for all $t \in\left[t_{1},+\infty\right)_{\mathrm{T}}$, $t_{1} \in\left[t_{0},+\infty\right)_{\mathrm{T}}$. We proceed as in the proof of Theorem 3.1 to obtain (3.8) and (3.9). Then from (3.9), we have for $t \geq u \geq T_{0}$

$$
\int_{u}^{t} c^{\lambda} H(t, s) \phi(s) \Psi(s) \Delta s-\int_{u}^{t} \frac{[|h(t, s)| \phi(\sigma(s)) A(\sigma(s))]^{\lambda+1}}{(\lambda+1)^{\lambda+1}[\phi(s) A(s) H(t, s)]^{\lambda}} \Delta s \leq H\left(t, t_{0}\right) w(u),
$$


thus,

$$
\begin{aligned}
& \limsup _{t \rightarrow+\infty} \frac{1}{H\left(t, t_{0}\right)} \int_{u}^{t} c^{\lambda} H(t, s) \phi(s) \Psi(s) \Delta s \\
& \quad \leq w(u)+\limsup _{t \rightarrow+\infty} \int_{u}^{t} \frac{[|h(t, s)| \phi(\sigma(s)) A(\sigma(s))]^{\lambda+1}}{(\lambda+1)^{\lambda+1}[\phi(s) A(s) H(t, s)]^{\lambda}} \Delta s .
\end{aligned}
$$

Then, in view of (3.11) and (3.12), from the above inequality, we have $c^{\lambda} \zeta_{1}(u) \leq w(u)+$ $\frac{\zeta_{2}(u)}{(\lambda+1)^{\lambda+1}}$, that is,

$$
\zeta_{1}(u)-\theta \zeta_{2}(u) \leq c^{-\lambda} w(u), \quad u \geq T_{0} \geq t_{0} .
$$

Moreover, from (3.8), we find that

$$
\begin{aligned}
& \frac{1}{H\left(t, t_{0}\right)} \int_{T_{0}}^{t}\left\{\frac{\lambda \phi(s) A(s) H(t, s)[w(\sigma(s))]^{(\lambda+1) / \lambda}}{[\phi(\sigma(s)) A(\sigma(s))]]^{(\lambda+1) / \lambda}}-|h(t, s)| w(\sigma(s))\right\} \Delta s \\
& \quad \leq w\left(T_{0}\right)-\frac{1}{H\left(t, t_{0}\right)} \int_{T_{0}}^{t} c^{\lambda} H(t, s) \phi(s) \Psi(s) \Delta s,
\end{aligned}
$$

this inequality implies

$$
\begin{aligned}
& \liminf _{t \rightarrow+\infty} \frac{1}{H\left(t, t_{0}\right)} \int_{T_{0}}^{t}\left\{\frac{\lambda \phi(s) A(s) H(t, s)[w(\sigma(s))]^{(\lambda+1) / \lambda}}{[\phi(\sigma(s)) A(\sigma(s))]^{(\lambda+1) / \lambda}}-|h(t, s)| w(\sigma(s))\right\} \Delta s \\
& \quad \leq C_{0},
\end{aligned}
$$

where $C_{0}=w\left(T_{0}\right)-c^{\lambda} \zeta_{1}\left(T_{0}\right)$ is a constant. Now, we claim that

$$
\liminf _{t \rightarrow+\infty} \frac{1}{H\left(t, t_{0}\right)} \int_{T_{0}}^{t} \frac{\lambda \phi(s) A(s) H(t, s)[w(\sigma(s))]^{(\lambda+1) / \lambda}}{[\phi(\sigma(s)) A(\sigma(s))]^{(\lambda+1) / \lambda}} \Delta s<+\infty
$$

In fact, if (3.16) does not hold, then there exists a sequence $\left\{T_{n}\right\}_{n=1}^{+\infty}: T_{n} \in\left[t_{2},+\infty\right)_{\mathrm{T}}$ with $\lim _{n \rightarrow+\infty} T_{n}=+\infty$ such that $\lim _{n \rightarrow+\infty} \frac{1}{H\left(T_{n}, t_{0}\right)} \int_{T_{0}}^{T_{n}} \frac{\lambda \phi(s) A(s) H\left(T_{n}, s\right)[w(\sigma(s))]^{(\lambda+1) / \lambda}}{[\phi(\sigma(s)) A(\sigma(s))]]^{(\lambda+1) / \lambda}} \Delta s=+\infty$, it follows from (3.15) that

$$
\lim _{n \rightarrow+\infty} \frac{1}{H\left(T_{n}, t_{0}\right)} \int_{T_{0}}^{T_{n}}\left|h\left(T_{n}, s\right)\right| w(\sigma(s)) \Delta s=+\infty
$$

Thus, for all sufficiently large positive integers $n$,

$$
\begin{gathered}
\frac{1}{H\left(T_{n}, t_{0}\right)} \int_{T_{0}}^{T_{n}} \frac{\left.\lambda \phi(s) A(s) H\left(T_{n}, s\right)[w(\sigma(s))]\right]^{(\lambda+1) / \lambda}}{[\phi(\sigma(s)) A(\sigma(s))]^{(\lambda+1) / \lambda}} \Delta s \\
-\frac{1}{H\left(T_{n}, t_{0}\right)} \int_{T_{0}}^{T_{n}}\left|h\left(T_{n}, s\right)\right| w(\sigma(s)) \Delta s<C_{0}+1 .
\end{gathered}
$$

Therefore, for all sufficiently large positive integers $n$ and $\varepsilon \in(0,1)$, we can easily find

$$
\frac{\int_{T_{0}}^{T_{n}}\left|h\left(T_{n}, s\right)\right| w(\sigma(s)) \Delta s}{\int_{T_{0}}^{T_{n}} \frac{\lambda \phi(s) A(s) H\left(T_{n}, s\right)[w(\sigma(s))]^{(\lambda+1) / \lambda}}{[\phi(\sigma(s)) A(\sigma(s))]^{(\lambda+1) / \lambda}} \Delta s}>1-\varepsilon>0 .
$$


On the other hand, by Lemma 2.5 , we obtain

$$
\begin{aligned}
\int_{T_{0}}^{T_{n}}\left|h\left(T_{n}, s\right)\right| w(\sigma(s)) \Delta s \\
=\lambda^{\frac{-\lambda}{\lambda+1}} \int_{T_{0}}^{T_{n}}\left[\frac{\lambda \phi(s) A(s) H\left(T_{n}, s\right)}{[\phi(\sigma(s)) A(\sigma(s))]^{(\lambda+1) / \lambda}}\right]^{\frac{\lambda}{\lambda+1}} w(\sigma(s)) \frac{\left|h\left(T_{n}, s\right)\right| \phi(\sigma(s)) A(\sigma(s))}{\left[\phi(s) A(s) H\left(T_{n}, s\right)\right]^{\lambda /(\lambda+1)} \Delta s} \\
\leq \lambda \frac{-\lambda}{\lambda+1}\left\{\int_{T_{0}}^{T_{n}} \frac{\lambda \phi(s) A(s) H\left(T_{n}, s\right)[w(\sigma(s))]^{(\lambda+1) / \lambda}}{[\phi(\sigma(s)) A(\sigma(s))]^{(\lambda+1) / \lambda}} \Delta s\right\}^{\frac{\lambda}{\lambda+1}} \\
\quad \times\left\{\int_{T_{0}}^{T_{n}} \frac{\left[\left|h\left(T_{n}, s\right)\right| \phi(\sigma(s)) A(\sigma(s))\right]^{\lambda+1}}{\left[\phi(s) A(s) H\left(T_{n}, s\right)\right]^{\lambda}} \Delta s\right\}^{\frac{1}{\lambda+1}} .
\end{aligned}
$$

Further, in view of (3.18), one can easily find

$$
\begin{aligned}
0 & <\frac{(1-\varepsilon)^{\lambda}}{H\left(T_{n}, t_{0}\right)} \int_{T_{0}}^{T_{n}}\left|h\left(T_{n}, s\right)\right| w(\sigma(s)) \Delta s \\
& <\frac{\left\{\int_{T_{0}}^{T_{n}}\left|h\left(T_{n}, s\right)\right| w(\sigma(s)) \Delta s\right\}^{\lambda+1}}{H\left(T_{n}, t_{0}\right)\left\{\int_{T_{0}}^{T_{n}} \frac{\lambda \phi(s) A(s) H\left(T_{n}, s\right)[w(\sigma(s))]^{(\lambda+1) / \lambda}}{[\phi(\sigma(s)) A(\sigma(s))]^{(\lambda+1) / \lambda}} \Delta s\right\}^{\lambda}} \\
& \leq \frac{\lambda^{-\lambda}}{H\left(T_{n}, t_{0}\right)} \int_{T_{0}}^{T_{n}} \frac{\left[\left|h\left(T_{n}, s\right)\right| \phi(\sigma(s)) A(\sigma(s))\right]^{\lambda+1}}{\left[\phi(s) A(s) H\left(T_{n}, s\right)\right]^{\lambda}} \Delta s \leq \lambda^{-\lambda} \zeta_{2}\left(T_{0}\right),
\end{aligned}
$$

contradicting (3.17). Therefore (3.16) holds. Now, in view of (3.14) and (3.16), it follows that

$$
\begin{aligned}
& \liminf _{n \rightarrow+\infty} \frac{1}{H\left(T_{n}, t_{0}\right)} \int_{T_{0}}^{T_{n}} \frac{H\left(T_{n}, s\right) \phi(s) A(s)\left[\zeta_{1}(\sigma(s))-\theta \zeta_{2}(\sigma(s))\right]_{+}^{(1+\lambda) / \lambda}}{[\phi(\sigma(s)) A(\sigma(s))]^{(\lambda+1) / \lambda}} \Delta s \\
& \quad \leq \liminf _{n \rightarrow+\infty} \frac{c^{-(\lambda+1)}}{H\left(T_{n}, t_{0}\right)} \int_{T_{0}}^{T_{n}} \frac{H\left(T_{n}, s\right) \phi(s) A(s)[w(\sigma(s))]^{(1+\lambda) / \lambda}}{[\phi(\sigma(s)) A(\sigma(s))]^{(\lambda+1) / \lambda}} \Delta s<+\infty .
\end{aligned}
$$

But this contradicts condition (3.13). The proof is complete.

Clearly, the following immediate result can be extracted from Theorem 3.3.

Theorem 3.4 Assume $\left(\mathrm{H}_{1}\right)-\left(\mathrm{H}_{4}\right)$ and (1.10). If there exist functions $\zeta_{1}(t), \zeta_{2}(t) \in C_{r d}(\mathbf{T}, \mathbf{R})$ and a constant $\omega \geq 1$ such that

$$
\begin{aligned}
& \limsup _{t \rightarrow+\infty} \frac{1}{t^{\omega}} \int_{u}^{t}(t-s)^{\omega} \Psi(s) \Delta s \geq \zeta_{1}(u), \\
& \limsup _{t \rightarrow+\infty} \frac{1}{t^{\omega}} \int_{u}^{t} \frac{(t-s)^{\omega}}{A^{\lambda}(s)}\left[\frac{\omega A(\sigma(s))}{t-s}+b(s)\right]^{\lambda+1} \Delta s \leq \zeta_{2}(u)
\end{aligned}
$$

for $u \geq T_{0}$, and

$$
\liminf _{t \rightarrow+\infty} \frac{1}{t^{\omega}} \int_{T_{0}}^{t} \frac{(t-s)^{\omega} A(s)\left[\xi_{1}(\sigma(s))-\theta \xi_{2}(\sigma(s))\right]_{+}^{(\lambda+1) / \lambda}}{[A(\sigma(s))]^{(\lambda+1) / \lambda}} \Delta s=+\infty
$$


for some constant $T_{0} \geq t_{0}$, where $\left[\zeta_{1}(\sigma(s))-\theta \zeta_{2}(\sigma(s))\right]_{+}=\max \left\{\left[\zeta_{1}(\sigma(s))-\theta \zeta_{2}(\sigma(s))\right], 0\right\}$, $\theta=c^{-\lambda}(\lambda+1)^{-(\lambda+1)}$, the constant $c$ is defined as in Lemma 2.2 , the function $\Psi(s)$ is defined as in Theorem 3.1, then (1.1) is oscillatory on $\left[t_{0},+\infty\right)_{\mathbf{T}}$.

Next, when (1.11) holds, we give some conditions that guarantee that every solution of (1.1) oscillates or converges to zero.

Theorem 3.5 Assume $\left(\mathrm{H}_{1}\right)-\left(\mathrm{H}_{4}\right)$, (1.11); if there exist $H \in \Omega$ and a positive and $\Delta$ differentiable function $\phi: \mathbf{T} \rightarrow \mathbf{R}$ such that (3.1) holds, and

$$
\int_{t_{2}}^{+\infty}\left[\frac{1}{A(t)} \int_{t_{2}}^{t} e_{-b / A}(t, \sigma(s)) \xi^{\lambda}(s) \Phi(s) \Delta s\right]^{1 / \lambda} \Delta t=+\infty
$$

for some constant $t_{2} \geq t_{0}$, where $\xi(t)=\int_{t}^{+\infty}\left[\frac{e_{-b / A}(s, t)}{A(s)}\right]^{1 / \lambda} \Delta s$, then (1.1) is oscillatory on $\left[t_{0},+\infty\right)_{\mathrm{T}}$

Proof Suppose to the contrary that $x(t)$ is a nonoscillatory solution of $(1.1)$ on $\left[t_{0},+\infty\right)_{\mathbf{T}}$. We may assume without loss of generality that $x(t)>0$ and $x(\tau(t))>0, x(\delta(t))>0$ for all $t \in$ $\left[t_{1},+\infty\right)_{\mathrm{T}}, t_{1} \in\left[t_{0},+\infty\right)_{\mathrm{T}}$, and then $y(t)>0$. Analogously, we shall distinguish the following two cases:

$$
\text { (I) } y^{\Delta}(t)>0 \quad \text { for } t \in\left[t_{1},+\infty\right)_{\mathrm{T}} ; \quad \text { (II) } y^{\Delta}(t)<0 \quad \text { for } t \in\left[t_{1},+\infty\right)_{\mathrm{T}} \text {. }
$$

Case (I) The proof when $y^{\Delta}(t)$ is eventually positive is similar to that of the proof of Theorem 3.1 and it hence is omitted.

Case (II) Since $y(t)>0, y^{\Delta}(t)<0$ for $t \in\left[t_{1},+\infty\right)_{\mathrm{T}}$ and $0<x(t) \leq y(t)$, in view of the definition of $y(t)$ and $0 \leq B(t)<1$, we have

$$
\lim _{t \rightarrow+\infty} x(t)=\lim _{t \rightarrow+\infty} x(\tau(t))=\lim _{t \rightarrow+\infty} x(\delta(t)) \geq \frac{1}{2} \lim _{t \rightarrow+\infty} y(t)>\frac{1}{3} \lim _{t \rightarrow+\infty} y(t),
$$

therefore, there exists $t_{2} \in\left[t_{1},+\infty\right)_{\mathrm{T}}$ such that $x(\delta(t)) \geq \frac{1}{3} y(t)$ for all $t \in\left[t_{2},+\infty\right)_{\mathrm{T}}$.

We proceed as in the proof of Lemma 2.6 to find that $\frac{\left.A(t)\left|y^{\Delta}(t)\right|\right|^{\lambda-1} y^{\Delta}(t)}{e_{-b / A}\left(t, t_{0}\right)}\left(t \in\left[t_{1},+\infty\right)_{\mathbf{T}}\right)$ is decreasing, and therefore, for all $s \geq t, s, t \in\left[t_{1},+\infty\right)_{\mathrm{T}}$, we have $\frac{A(s)\left|y^{\Delta}(s)\right|^{\lambda-1} y^{\Delta}(s)}{e_{-b / A}\left(s, t_{0}\right)} \leq$ $\frac{A(t)\left|y^{\Delta}(t)\right|^{\lambda-1} y^{\Delta}(t)}{e_{-b / A}\left(t, t_{0}\right)}$, i.e., $\left[-y^{\Delta}(s)\right]^{\lambda} \geq A(t)\left[-y^{\Delta}(t)\right]^{\lambda} \frac{e_{-b / A}(s, t)}{A(s)}$, and thus, $y^{\Delta}(s) \leq[A(t)]^{\frac{1}{\lambda}} y^{\Delta}(t) \times$ $\left[\frac{e_{-} b / A(s, t)}{A(s)}\right]^{1 / \lambda}$, it follows that

$$
y(u)-y(t) \leq[A(t)]^{\frac{1}{\lambda}} y^{\Delta}(t) \int_{t}^{u}\left[\frac{e_{-b / A}(s, t)}{A(s)}\right]^{1 / \lambda} \Delta s .
$$

Now, letting $u \rightarrow+\infty$, then we get (in view of Lemma 2.3, we see that $A(t)\left|y^{\Delta}(t)\right|^{\lambda-1} y^{\Delta}(t)$ $\left(t \in\left[t_{1},+\infty\right)_{\mathrm{T}}\right)$ is decreasing as well)

$$
\begin{aligned}
y(t) & \geq-[A(t)]^{\frac{1}{\lambda}} y^{\Delta}(t) \int_{t}^{+\infty}\left[\frac{e_{-b / A}(s, t)}{A(s)}\right]^{1 / \lambda} \Delta s=-\xi(t)[A(t)]^{\frac{1}{\lambda}} y^{\Delta}(t) \\
& \geq-\xi(t)\left[A\left(t_{1}\right)\right]^{\frac{1}{\lambda}} y^{\Delta}\left(t_{1}\right)=b \xi(t),
\end{aligned}
$$


where $b_{0}=\left[A\left(t_{1}\right)\right]^{1 / \lambda}\left[-y^{\Delta}\left(t_{1}\right)\right]>0$ is a constant. Consequently, by (2.2), we find

$$
\begin{aligned}
-\left[A(t) \varphi\left(y^{\Delta}(t)\right)\right]^{\Delta} & \geq b(t) \varphi\left(y^{\Delta}(t)\right)+\Phi(t)[x(\delta(t))]^{\lambda} \geq b(t) \varphi\left(y^{\Delta}(t)\right)+\frac{1}{3^{\lambda}} \Phi(t)[y(t)]^{\lambda} \\
& \geq b(t) \varphi\left(y^{\Delta}(t)\right)+\frac{b_{0}^{\lambda}}{3^{\lambda}} \Phi(t) \xi^{\lambda}(t) .
\end{aligned}
$$

Define the function $U(t)=A(t) \varphi\left(y^{\Delta}(t)\right)=A(t)\left|y^{\Delta}(t)\right|^{\lambda-1} y^{\Delta}(t)=-A(t)\left|y^{\Delta}(t)\right|^{\lambda}$, with (3.23) this yields

$$
U^{\Delta}(t) \leq-\frac{b(t)}{A(t)} U(t)-\frac{b_{0}^{\lambda}}{3^{\lambda}} \Phi(t) \xi^{\lambda}(t), \quad t \in\left[t_{2},+\infty\right)_{\mathbf{T}} .
$$

The inequality in (3.24) is the assumed inequality of [2, Theorem 6.1]. All other assumptions of [2, Theorem 6.1], e.g., $-b / A \in \Re^{+}$are satisfied as well. Hence the conclusion of [2, Theorem 6.1] holds, i.e.,

$$
\begin{aligned}
U(t) & \leq U\left(t_{2}\right) e_{-b / A}\left(t, t_{2}\right)-\frac{b_{0}^{\lambda}}{3^{\lambda}} \int_{t_{2}}^{t} e_{-b / A}(t, \sigma(s)) \xi^{\lambda}(s) \Phi(s) \Delta s \\
& <-\frac{b_{0}^{\lambda}}{3^{\lambda}} \int_{t_{2}}^{t} e_{-b / A}(t, \sigma(s)) \xi^{\lambda}(s) \Phi(s) \Delta s,
\end{aligned}
$$

for all $t \in\left[t_{2},+\infty\right)_{\mathrm{T}}$, i.e., $y^{\Delta}(t)<-\frac{b_{0}}{3}\left[\frac{1}{A(t)} \int_{t_{2}}^{t} e_{-b / A}(t, \sigma(s)) \xi^{\lambda}(s) \Phi(s) \Delta s\right]^{1 / \lambda}$, and thus,

$$
y(u)<y\left(t_{2}\right)-\frac{b_{0}}{3} \int_{t_{2}}^{u}\left[\frac{1}{A(t)} \int_{t_{2}}^{t} e_{-b / A}(t, \sigma(s)) \xi^{\lambda}(s) \Phi(s) \Delta s\right]^{1 / \lambda} \Delta t \rightarrow-\infty \quad \text { as } u \rightarrow+\infty,
$$

which contradicts with $y(t)>0$. This completes the proof.

Using the same method as in the proof of Theorem 3.5, we can now obtain the following results.

Theorem 3.6 Assume $\left(\mathrm{H}_{1}\right)-\left(\mathrm{H}_{4}\right),(1.11)$ and (3.22) hold. If there exists a positive and $\Delta$ differentiable function $\phi: \mathbf{T} \rightarrow \mathbf{R}$ and $\omega \geq 1$ such that (3.10) holds, then (1.1) is oscillatory on $\left[t_{0},+\infty\right)_{\mathrm{T}}$.

Theorem 3.7 Assume $\left(\mathrm{H}_{1}\right)-\left(\mathrm{H}_{4}\right),(1.11)$, and (3.22) hold. If there exist functions $H \in \Omega$, $\zeta_{1}(t), \zeta_{2}(t) \in C_{r d}(\mathbf{T}, \mathbf{R})$, and a positive, nondecreasing, differentiable function $\phi \in C_{r d}\left(\mathbf{T}, \mathbf{R}^{+}\right)$ such that (3.11)-(3.13) hold, then (1.1) is oscillatory on $\left[t_{0},+\infty\right)_{\mathbf{T}}$.

Theorem 3.8 Assume $\left(\mathrm{H}_{1}\right)-\left(\mathrm{H}_{4}\right)$, (1.11), and (3.22) hold. If there exist functions $\zeta_{1}(t), \zeta_{2}(t) \in$ $C_{r d}(\mathbf{T}, \mathbf{R})$ and a constant $\omega \geq 1$ such that (3.19)-(3.21) hold, then (1.1) is oscillatory on $\left[t_{0},+\infty\right)_{\mathbf{T}}$

Remark 3.2 Our results in this paper not only extend and improve some known results, and show some results of $[10-17,21,22,25]$ to be special examples of our results, but also unify the oscillation of the second-order nonlinear delay damped differential equations (1.2) and the second-order nonlinear delay damped difference equations (1.3). The theorems in this paper are new even for the cases $\mathbf{T}=\mathbf{R}$ and $\mathbf{T}=\mathbf{Z}$. 


\section{Examples}

In this section, we give several examples to illustrate our results.

Example 4.1 Consider second-order delay dynamic equation on time scales:

$$
\left\{A(t) \varphi\left(\left[x(t)+\left(\frac{1}{\sqrt{2}}-\frac{1}{t}\right) x(\tau(t))\right]^{\Delta}\right)\right\}^{\Delta}+P(t) \varphi(x(\delta(t)))=0, \quad t \in \mathbf{T}, t \geq t_{0}
$$

here $b(t) \equiv 0, Q(t) \equiv 0, B(t)=\frac{1}{2}-\frac{1}{2 t}, F(u)=u$. Pick $A(t)=t^{\frac{2}{3}}, \lambda=\frac{5}{3}, \tau(t)=\delta(t)=\frac{t}{2}, P(t)=$ $\frac{1}{2 t}\left(\frac{2 t}{t+2}\right)^{\frac{5}{3}}, \mathbf{T}=2^{\mathbf{Z}}, t_{0}=2$, then (4.1) becomes a second-order variable delay 2-difference equation. We have

$$
\begin{aligned}
& \Phi(t)=L P(t)-\eta Q(t)=\frac{1}{2 t}\left(\frac{2 t}{t+2 \sqrt{2}}\right)^{\frac{5}{3}}>0 \\
& \int_{t_{0}}^{t}\left[\frac{e_{-b / A}\left(s, t_{0}\right)}{A(s)}\right]^{\frac{1}{\lambda}} \Delta s=\int_{2}^{t} s^{-\frac{2}{5}} \Delta s=\frac{t^{\frac{3}{5}}-2^{\frac{3}{5}}}{2^{\frac{3}{5}}-1} \rightarrow+\infty \quad \text { as } t \rightarrow+\infty
\end{aligned}
$$

Hence, conditions $\left(\mathrm{H}_{1}\right)-\left(\mathrm{H}_{4}\right)$ and (1.10) are clearly satisfied. Now, in Theorem 3.2, pick $\phi(t)=1, \omega=2$. Then for all $t \geq 2$, we can obtain

$$
\begin{aligned}
& \limsup _{t \rightarrow+\infty} \frac{1}{t^{\omega}} \int_{T_{0}}^{t}(t-s)^{\omega}\left\{c^{\lambda} \phi(s) \Psi(s)-\frac{(\lambda+1)^{-(\lambda+1)}}{[\phi(s) A(s)]^{\lambda}}\left[\left|\phi^{\Delta}(s)-\frac{\phi(s) b(s)}{A(\sigma(s))}\right| A(\sigma(s))\right]^{\lambda+1}\right\} \Delta s \\
& \quad=\limsup _{t \rightarrow+\infty} \frac{1}{t^{2}} \int_{2}^{t}(t-s)^{2}\left\{c^{\frac{5}{3}} \frac{1}{2 s}\left(\frac{2 s}{s+2}\right)^{\frac{5}{3}}\left[1-\left(\frac{1}{2}-\frac{1}{s}\right)\right]^{\frac{5}{3}}\left[\frac{1}{\sqrt{2}}\right]^{\frac{5}{3}}-0\right\} \Delta s \\
& =\left[\frac{c}{\sqrt{2}}\right]^{\frac{5}{3}} \limsup _{t \rightarrow+\infty} \frac{1}{t^{2}} \int_{2}^{t} \frac{(t-s)^{2}}{2 s} \Delta s \\
& =\left[\frac{c}{\sqrt{2}}\right]^{\frac{5}{3}} \limsup _{t \rightarrow+\infty}\left\{\frac{1}{2}\left[\log _{2}(t)-1\right]+\frac{1}{2 t^{2}}\left(\frac{t^{2}-2^{2}}{2^{2}-1}\right)-\frac{1}{t}(t-2)\right\}=+\infty
\end{aligned}
$$

Therefore, by Theorem 3.2, (4.1) is oscillatory.

Example 4.2 Consider second-order nonlinear variable delay dynamic equation on time scales T:

$$
\left[t^{\frac{2}{5}} x^{\Delta}(t)\right]^{\Delta}+t^{-\frac{13}{5}} x^{\Delta}(t)+t^{-\frac{11}{10}} x\left(\frac{t}{2}\right)=0, \quad t \in \mathbf{T}=2^{\mathbf{Z}}, t \geq t_{0}:=2
$$

here $A(t)=t^{2 / 5}, B(t) \equiv 0, b(t)=t^{-13 / 5}, P(t)=t^{-11 / 10}, Q(t) \equiv 0, F(u)=u, \delta(t)=t / 2, \lambda=1$. Similarly, it is easy to see that conditions $\left(\mathrm{H}_{1}\right)-\left(\mathrm{H}_{4}\right)$ and (1.10) are satisfied. Now, take $\omega=2$, $\phi(t)=1$, then we have

$$
\begin{aligned}
& \limsup _{t \rightarrow+\infty} \frac{1}{t^{\omega}} \int_{T_{0}}^{t}(t-s)^{\omega}\left\{c^{\lambda} \phi(s) \Psi(s)-\frac{(\lambda+1)^{-(\lambda+1)}}{[\phi(s) A(s)]^{\lambda}}\left[\left|\phi^{\Delta}(s)-\frac{\phi(s) b(s)}{A(\sigma(s))}\right| A(\sigma(s))\right]^{\lambda+1}\right\} \Delta s \\
& \quad=\limsup _{t \rightarrow+\infty} \frac{1}{t^{2}} \int_{2}^{t}(t-s)^{2}\left\{c \frac{2^{-2}}{s^{11 / 10}}-\frac{2^{-2}}{s^{2 / 5}}\left(s^{-13 / 5}\right)^{2}\right\} \Delta s \\
& \quad=\frac{1}{4} \limsup _{t \rightarrow+\infty} \frac{1}{t^{2}} \int_{2}^{t}(t-s)^{2}\left(\frac{c}{s^{11 / 10}}-\frac{1}{s^{28 / 5}}\right) \Delta s<+\infty
\end{aligned}
$$


which implies that (3.10) does not hold. Therefore, Theorem 3.1 and Theorem 3.2 cannot be applied to (4.2), and one can easily see that the results in $[10-17,21,22,25]$ cannot be applied in (4.2).

Next, we will apply Theorem 3.4 and it remains to satisfy the conditions (3.19)-(3.21).

Since

$$
\begin{aligned}
& \limsup _{t \rightarrow+\infty} \frac{1}{t^{\omega}} \int_{u}^{t}(t-s)^{\omega} \Psi(s) \Delta s \\
& \quad=\limsup _{t \rightarrow+\infty} \frac{1}{t^{2}} \int_{u}^{t}(t-s)^{2} \frac{2^{-2}}{s^{11 / 10}} \Delta s=\frac{1}{4} \limsup _{t \rightarrow+\infty} \frac{1}{t^{2}} \int_{u}^{t}\left(t^{2} s^{-11 / 10}-2 t s^{-1 / 10}+s^{9 / 10}\right) \Delta s \\
& \quad=\frac{1}{4} \frac{1}{u^{1 / 10}-1} \geq \frac{1}{4 u^{1 / 10}}=\xi_{1}(u), \\
& \limsup _{t \rightarrow+\infty} \frac{1}{t^{\omega}} \int_{u}^{t} \frac{(t-s)^{\omega}}{A^{\lambda}(s)}\left[\frac{\omega A(\sigma(s))}{t-s}+b(s)\right]^{\lambda+1} \Delta s \\
& \quad=\limsup _{t \rightarrow+\infty} \frac{1}{t^{2}} \int_{u}^{t} \frac{1}{s^{2 / 5}}\left[2(2 s)^{2 / 5}+(t-s) s^{-13 / 5}\right]^{2} \Delta s \\
& \quad=\limsup _{t \rightarrow+\infty} \frac{1}{t^{2}} \int_{u}^{t}\left[2^{\frac{14}{5}} s^{\frac{2}{5}}+2^{\frac{12}{5}} t s^{-\frac{13}{5}}-2^{\frac{12}{5}} s^{-\frac{8}{5}}+t^{2} s^{-\frac{28}{5}}-2 t s^{-\frac{23}{5}}+s^{-\frac{18}{5}}\right] \Delta s \\
& \quad=\frac{u^{-23 / 5}}{1-u^{-23 / 5}} \leq \frac{u^{-23 / 5}}{1-2^{-23 / 5}}=\xi_{2}(u),
\end{aligned}
$$

we have

$$
\xi_{1}(\sigma(s))-\theta \xi_{2}(\sigma(s))=\frac{1}{4(2 s)^{1 / 10}}-\theta \frac{(2 s)^{-23 / 5}}{1-2^{-23 / 5}}=\frac{k_{1}}{s^{1 / 10}}-\frac{k_{2}}{s^{23 / 5}},
$$

where $k_{1}=\frac{1}{4 \cdot 2^{1 / 10}}, k_{2}=\frac{2^{-23 / 5}}{1-2^{-23 / 5}} \theta$. We conclude

$$
\begin{aligned}
& \liminf _{t \rightarrow+\infty} \frac{1}{t^{\omega}} \int_{T_{0}}^{t} \frac{(t-s)^{\omega} A(s)\left[\xi_{1}(\sigma(s))-\theta \xi_{2}(\sigma(s))\right]_{+}^{(\lambda+1) / \lambda}}{[A(\sigma(s))]^{(\lambda+1) / \lambda}} \Delta s \\
& \quad=\liminf _{t \rightarrow+\infty} \frac{1}{t^{2}} \int_{2}^{t} \frac{(t-s)^{2} s^{2 / 5}\left(\frac{k_{1}}{s^{1 / 10}}-\frac{k_{2}}{s^{23 / 5}}\right)^{2}}{\left[(2 s)^{2 / 5}\right]^{2}} \Delta s \\
& =\frac{1}{2^{4 / 5}} \liminf _{t \rightarrow+\infty} \frac{1}{t^{2}} \int_{2}^{t}(t-s)^{2}\left(\frac{k_{1}^{2}}{s^{3 / 5}}-2 \frac{k_{1} k_{2}}{s^{51 / 10}}+\frac{k_{2}^{2}}{s^{48 / 5}}\right) \Delta s=+\infty .
\end{aligned}
$$

So, (3.19)-(3.21) are satisfied as well. Altogether, by Theorem 3.4, we find that (4.2) is oscillatory.

Remark 4.1 One can easily see that the recent results cannot be applied in (1.1) or (4.1) and (4.2), so our results are new ones. 


\section{Acknowledgements}

Supported by the Scientific Research Fund of Education Department of Guangxi Zhuang Autonomous Region (2013YB223) and Hunan Province Science and Technology Project (2012FJ3107)

Received: 12 October 2014 Accepted: 19 December 2014 Published online: 26 March 2015

\section{References}

1. Hilger, S: Analysis on measure chains - a unified approach to continuous and discrete calculus. Results Math. 18 18-56 (1990)

2. Agarwal, RP, Grace, SR, O’Regan, D: Oscillation Theory for Second Order Linear, Half-Linear, Superlinear and Sublinear Dynamic Equations. Kluwer, Dordrecht (2002)

3. Agarwal, RP, Bohner, M, Grace, SR, O'Regan, D: Discrete Oscillation Theory. Hindawi Publishing Corporation, New York (2005)

4. Bohner, M, Peterson, A: Dynamic Equations on Time Scales: An Introduction with Applications. Birkhauser, Boston (2001)

5. Bohner, M, Peterson, A: Advances in Dynamic Equations on Time Scales. Birkhauser, Boston (2003)

6. Agarwal, RP, Bohner, M, O'Regan, D, Peterson, A: Dynamic equations on time scales: a survey. J. Comput. Appl. Math. 141, 1-26 (2002)

7. Bohner, M, Saker, SH: Oscillation of second order nonlinear dynamic equations on time scales. Rocky Mt. J. Math. 34 1239-1254 (2004)

8. Erbe, L: Oscillation criteria for second order linear equations on a time scale. Can. Appl. Math. Q. 9, 345-375 (2001)

9. Erbe, L, Peterson, A, Rehák, P: Comparison theorems for linear dynamic equations on time scales. J. Math. Anal. Appl. $275,418-438(2002)$

10. Sun, S, Han, Z, Zhang, C: Oscillation of second order delay dynamic equations on time scales. J. Appl. Math. Comput. 30, 459-468 (2009)

11. Grace, SR, Agarwal, RP, Kaymakcalan, B, Sae-jie, W: Oscillation theorems for second order nonlinear dynamic equations. J. Appl. Math. Comput. 32, 205-218 (2010)

12. Agarwal, RP, Bohner, M, Saker, SH: Oscillation of second order delay dynamic equations. Can. Appl. Math. Q. 13, 1-18 (2005)

13. Sahiner, Y: Oscillation of second order delay differential equations on time scales. Nonlinear Anal. TMA 63, 1073-1080 (2005)

14. Saker, SH: Oscillation criteria of second-order half-linear dynamic equations on time scales. J. Comput. Appl. Math 177, 375-387 (2005)

15. Erbe, L, Peterson, A, Saker, SH: Oscillation criteria for second order nonlinear delay dynamic equations. J. Math. Anal. Appl. 333, 505-522 (2007)

16. Erbe, L, Hassan, TS, Peterson, A: Oscillation criteria for nonlinear functional neutral dynamic equations on time scales. J. Differ. Equ. Appl. 15(11-12), 1097-1116 (2009)

17. Grace, SR, Bohner, M, Agarwal, RP: On the oscillation of second-order half-linear dynamic equations. J. Differ. Equ. Appl. 15(5), 451-460 (2009)

18. Agarwal, RP, Bohner, M, Li, WT: Nonoscillation and Oscillation: Theory for Functional Differential Equations. Marcel Dekker, New York (2004)

19. Tuna, A, Kutukcu, S: Some integral inequalities on time scales. Appl. Math. Mech. 29(1), 23-29 (2008)

20. Bohner, M: Some oscillation criteria for first order delay dynamic equations. Far East J. Appl. Math. 18(3), 289-304 (2005)

21. Zhang, QX, Gao, L: Oscillation criteria for second-order half-linear delay dynamic equations with damping on time scales. Sci. Sin., Math. 40(7), 673-682 (2010)

22. Zhang, QX: Oscillation of second-order half-linear delay dynamic equations with damping on time scales. J. Comput. Appl. Math. 235, 1180-1188 (2011)

23. Han, ZL, Li, TX, Sun, SR, et al.: Oscillation behavior of solutions of third-order nonlinear delay dynamic equations on time scales. Commun. Korean Math. Soc. 26(3), 499-513 (2011)

24. Chen, DX, Qu, PX, Lan, YH: Oscillation of second-order nonlinear dynamic equations with positive and negative coefficients. Adv. Differ. Equ. 2013, 168 (2013). doi:10.1186/1687-1847-2013-168

25. Zhang, QX, Gao, L, Wang, L: Oscillation of second-order nonlinear delay dynamic equations on time scales. Comput. Math. Appl. 61, 2342-2348 (2011)

26. Han, ZL, Li, TX, Sun, SR, Sun, YB: Remarks on the paper [Appl. Math. Comput. 206, 388-396 (2009)]. Appl. Math. Comput. 215, 3998-4007 (2010)

27. Yang, JS: Oscillation criteria for certain third-order delay dynamic equations. Adv. Differ. Equ. 2013, 178 (2013)

28. Han, ZL, Sun, YB, Xu, MR: Oscillation criteria for $p$-Laplacian delay dynamic equations with damping on time scales. Adv. Differ. Equ. 2013, 145 (2013) 